\title{
STATISTICAL EVALUATION AND CORRELATIVE STUDY OF NEOPLASTIC AND NON-NEOPLASTIC LESIONS OF OESOPHAGUS
}

\author{
Kavitha Duraisamy1, Revathi Ramakrishnan²
}

${ }^{1}$ Associate Professor, Department of Pathology, Government Theni Medical College, Theni.

${ }^{2}$ Associate Professor, Department of Pathology, Government Theni Medical College, Theni.

ABSTRACT
BACKGROUND
Oesophageal lesions once thought to be rare is nowadays being one of the common disorder affecting the people throughout the
world. The clinical, endoscopic findings and histopathologic changes of oesophageal mucosa induced by Gastro-oesophageal Reflux
Disease (GERD) has been mainly focused and analysed.

\section{MATERIALS AND METHODS}

Patients presented with symptoms and signs of oesophageal lesions during January 2000 to September 2005 were included in this study. Upper gastrointestinal endoscopy was performed. Endoscopic changes were noted in the oesophagus and stomach. In oesophagectomy specimens, four longitudinal sections were taken, one including a portion of non-neoplastic mucosa proximal to tumour and another distal to the tumour. Sections taken from biopsy and resected specimens that were fixed in $10 \%$ buffered formalin were cut. The sections were stained with haematoxylin and eosin for evaluation of histopathologic features, Alcian blue (AB), Periodic Acid-Schiff stain (PAS) to demonstrate metaplasia.

\section{OBSERVATION AND RESULTS}

This study covered a total of 323 cases, in which 277 were endoscopic biopsies and 46 were oesophagectomy specimens. In 277 endoscopic biopsies, 193 were males (69.68) and 84 were females (30.33\%). There was increased incidence of oesophageal lesions observed. In the age group of 51-60, most of the patients in our study had complained of dysphagia (90.25\%) followed by loss of weight (70.04\%) and anorexia (54.87\%). Among the 277 cases, 9 cases were Barrett's (3.24\%), 18 cases were diagnosed as adenocarcinoma (6.498\%), 176 cases were diagnosed as squamous cell carcinomas (63.18\%), 42 cases were squamous intraepithelial lesion (15.16\%), 14 cases were interpreted as normal stratified squamous epithelium (5.05\%), 14 cases were interpreted as only necrotic material/no tissue (5.05\%). One case of basaloid squamous cell carcinoma, which is of poor prognosis was seen. One case of adenosquamous carcinoma characterised by mixed glandular and squamous differentiation is a rare aggressive neoplasm. One case of primary malignant melanoma, which on immunohistochemistry showed positivity for S100.

\section{CONCLUSION}

This present study is to evaluate the incidence of neoplastic and non-neoplastic oesophageal lesions, site of occurrence of oesophageal carcinomas, the incidence of Barrett's oesophagus, and subsequently adenocarcinoma with mucin histochemistry and to evaluate the role of immunohistochemistry in doubtful cases of oesophagectomy and endoscopic biopsy specimens.

\section{KEYWORDS}

Oesophageal Biopsy, Barrett's, Histopathology.

HOW TO CITE THIS ARTICLE: Duraisamy K, Ramakrishnan R. Statistical evaluation and correlative study of neoplastic and nonneoplastic lesions of oesophagus. Evolution Med. Dent. Sci. 2016;5(63):4480-4487, DOI: 10.14260/jemds/2016/1021

\section{INTRODUCTION}

Oesophageal lesions once thought to be rare is nowadays being one of the common disorder affecting the people throughout the world and one among the top ten malignant affecting the people of Tamilnadu ranking fourth in the gastrointestinal tract following stomach, colon and pancreas.

Twenty percent of oesophageal tumours are benign, mostly leiomyoma remaining $80 \%$ are malignant tumours, in which about $90 \%$ are squamous cell carcinoma.

In this study the patients presenting with dysphagia, loss of weight, anorexia and regurgitation of food is undertaken to

Financial or Other, Competing Interest: None.

Submission 15-07-2016, Peer Review 28-07-2016,

Acceptance 30-07-2016, Published 08-07-2016.

Corresponding Author:

Dr. Revathi Ramakrishnan,

Door No. 1-1-93/F10, Usilai Road,

Peraiyur, Madurai-625703, Tamilnadu.

E-mail: revathiram67@gmail.com

DOI: $10.14260 /$ jemds/2016/1021 evaluate the relationship of this symptom complex to underlying oesophageal lesions with special reference to Barrett's oesophagus.

The clinical, endoscopic findings and histopathologic changes of oesophageal mucosa induced by Gastrooesophageal Reflux Disease (GERD) has been mainly focused and analysed.

The oesophagectomy specimens subjected for histopathological evaluation are also concentrated towards the histological type, morphological variants, presence or absence of changes in the upper gastric region and subsequent dysplastic changes at the adjacent mucosa.

In the present study, the histopathological features of Barrett's oesophagus is in detail paying particular attention to the presence of goblet cells. In addition, the recent literature regarding epidemiology, clinical features, pathogenesis and pathology of oesophageal lesions is reviewed. 


\section{Oesophagectomy and Endoscopic Biopsy Specimens were} Studied to find out the

1. Role of socioeconomic status, epidemiology and living conditions in the occurrence of oesophageal carcinomas.

2. Incidence of neoplastic and non-neoplastic oesophageal lesions.

3. To evaluate site of occurrence of oesophageal carcinomas.

4. To study the incidence of Barrett's oesophagus and subsequently adenocarcinoma with mucin histochemistry.

5. To evaluate the role of immunohistochemistry in doubtful cases.

\section{MATERIALS AND METHODS}

Patients presented with symptoms and signs of oesophageal lesions during January 2000 to September 2005 were included in this study.

A thorough clinical evaluation, blood count, urine examination, Barium swallow and ultrasound abdomen (in proportion of cases) were done in each case. A detailed history with particular attention to socioeconomic status, housing conditions, nutrition, alcoholism, smoking, tobacco and betel nut chewing and history of upper gastrointestinal complaints in other family members were also recorded.

\section{ENDOSCOPY}

Upper gastrointestinal endoscopy was performed with Pentax video endoscopy system 1998 E 3300 with non-spiked Pentax biopsy forceps applied with Xylocaine jelly at the tip. Endoscopic changes were noted in the oesophagus and stomach.

\section{Histologic Study of Oesophageal Biopsy and Resected Specimens}

Two biopsy specimens were taken from the oesophagus, which were immediately fixed in $10 \%$ buffered neutral formalin for histological evaluation.

In oesophagectomy specimens, the resected area was opened longitudinally from one end to another in the fresh state itself on the side opposite the tumour. The lesional area was three portions as adjacent, proximal and distal to the tumour. Specimens were pinned in a cork board with mucosal side up allowed to float in formalin container for overnight fixation. Four longitudinal sections were taken, one including a portion of non-neoplastic mucosa proximal to tumour and another distal tumour. Two sections from the cardiac end of stomach, one including the gastro-oesophageal junction was taken. Proximal resected margin, distal resected margin, lymph nodes if present were also sectioned and processed. Sections taken from biopsy and resected specimens that were fixed in $10 \%$ buffered formalin were cut. The sections were stained with Haematoxylin and Eosin for evaluation of histopathologic features, Alcian Blue (AB), Periodic Acid-Schiff Stain (PAS) to demonstrate metaplasia.

Oesophageal lesions were defined and classified according to the established histological criteria as per Sternberg's Diagnostic Surgical Pathology. The presence of oesophagitis, dysplastic changes. Barrett's oesophagus with intestinal metaplasia, benign and malignant neoplasm were recorded in all cases of oesophageal biopsies. The most prevalent appearance in each slide was matched with standard panel, formulated by renowned authors that resembles it most closely.

The presence or absence of other histological features like fungal infections, candida, congenital abnormalities, erosions and nonspecific oesophageal changes were also carefully looked for, paying particular attention to histomorphological alteration.

Histochemistry with Alcian Blue with PAS was performed to demonstrate the presence of goblet cells in Barrett's oesophagus.

In two doubtful cases, immunohistochemistry with markers SI00, Vimentin, EMA, cytokeratin was also done.

\section{OBSERVATION AND RESULTS}

This study covered a total of 323 cases, in which 277 were endoscopic biopsies and 46 were oesophagectomy specimens.

In 277 endoscopic biopsies, 193 were males (69.68\%) with age ranging from 20 to 80 yrs. (Mean age 50 yrs.), 84 were females (30.33\%) with age ranging from 20 to $80 \mathrm{yrs}$. (Mean age 50 yrs.).

Table No. 1 shows the total number of oesophageal biopsy specimens referred for histological evaluation during the period January 2000 - September 2005. The average incidence is $9.75 \%$. Table No. $1 \mathrm{~A}$ shows the incidence of oesophageal specimens received after initial endoscopic evaluation for management during the period from 2000 to 2005. The average incidence is $0.3 \%$.

\section{Age Incidence}

The patients clinically manifested with dysphagia, weight loss, nausea and vomiting who were further biopsied were divided into 6 groups according to their age [i.e. 20-30 yrs., 31-40 yrs., 41-50 yrs., 51-60 yrs., 61-70 yrs., 71-80 yrs.]. There was increased incidence of oesophageal lesions observed in the age group of 51-60 yrs. (33.21\%) followed by $41-50$ yrs. $(24.55 \%)$ and $61-70$ yrs. (14.49\%). The age distribution of oesophageal biopsies is given in Table No. II. Similarly, the age distribution of oesophageal specimens received were also calculated accordingly as given in Table IIA. Most of the patients belonged to lower socioeconomic status lived in overcrowded surroundings. Table No. III shows the distribution of cases according to their clinical presentation in endoscopic biopsies. Most of the patients in our study had complained of dysphagia $(90.25 \%)$ followed by loss of weight $(70.04 \%)$ and anorexia (54.87\%).

\section{Oesophageal Biopsy Evaluation}

277 cases of oesophageal biopsy were received and histopathological evaluation was done. Among the 277 cases, 9 cases were Barrett's (3.24\%), 18 cases were diagnosed as adenocarcinoma (Fig. 1 and 2) (6.498\%), 176 cases were diagnosed as squamous cell carcinomas (63.18\%), 42 cases were squamous intraepithelial lesion (Fig. 10) (15.16\%), 14 cases were interpreted as normal stratified squamous epithelium (5.05\%), 14 cases were interpreted as only necrotic material/no tissue $(5.05 \%)$. Table No. IV shows the incidence of lesions and its percentage.

Table No. IV shows Squamous Cell Carcinoma (SCC) of the oesophagus is the commonest malignant neoplasm [176 cases, (63.54\%) followed by SIL [42 cases, (15.16\%)]. Incidence of adenocarcinoma oesophagus is only $6.49 \%$ Table IV-A and Table IV-B shows oesophageal biopsies. Squamous cell 
carcinoma was the commonest neoplasm (Males 9.75\%, Females 5.42\%).

Incidence of Barrett's oesophagus characterised by metaplastic columnar epithelium intentional type with goblet cells that has replaced the original squamous lined mucosa is also observed. In males, 7 cases of BE $(2.53 \%)$ was observed and 16 cases of adenocarcinoma (5.78\%) was seen.

Table IV A and B also clearly shows the oesophageal lesions (neoplastic, preneoplastic) are common in males when compared with females and the incidence of adenocarcinoma is much low when compared with classical SCC. Among the 277 cases, one case of squamous papilloma which occurred in a 50 yrs. male with characteristic features of central core of connective tissue covered with hyperplastic squamous cells in an orderly arrangement and without dysplastic changes was observed. One case of fibromuscular polyp composed of mesenchymal tissue, which was fibrous and covered by intact squamous epithelium occurred in 38 yrs. males. One case of adenosquamous carcinoma characterised by proliferation of malignant glands adjacent to malignant squamous epithelium was observed in 70 yrs. male.

One case of malignant melanoma (Fig. 5) characterised by small, dark, flat lesions in the upper GI endoscopy which revealed aggregates of pleomorphic cells with hyperchromatic nuclei, binucleated cells and intracellular melanin pigmentation was observed in 60 yrs. female. One case of sarcomatoid carcinoma (Fig. 3, Fig. 4) with features of both spindle cells and squamous cell carcinoma components was observed in 55 yrs. male.

\section{Histopathological Evaluation}

46 oesophagectomy specimens were received, of which 41 cases $(89.13 \%)$ were squamous cell carcinoma and 5 cases were adenocarcinoma (10.86\%). The received specimens were categorised according to the site of presentation whether in upper $1 / 3^{\text {rd }}$, middle $1 / 3^{\text {rd }}$ and lower $1 / 3^{\text {rd }}$ and also categorised according to the morphological appearance. Table No. V shows the distribution of cases according to the morphological appearance. Most of the presented cases were in advanced stage with exophytic, fungating growth on gross appearance (Fig. 11). Most of the cases presented in lower $1 / 3^{\text {rd }}$ segment of oesophagus (34 cases, $73.91 \%$ ). Relative distribution of squamous cell carcinoma according to site is shown in Table No. V-A. Table No. V-B shows the relative distribution of squamous cell carcinoma in both sexes. Majority of patients who had squamous cell carcinoma were males (60.97\%). Table No. V-C shows the relative distribution of squamous cell carcinoma according to differentiation. Most of the adenocarcinoma occurred in males. Table No. VI shows the relative distribution of adenocarcinoma according to differentiation.

Most of the squamous cell carcinoma were well differentiated ( 26 cases $-63.41 \%$ ); 13 cases of squamous cell carcinoma were moderately differentiated (13 cases, $31.71 \%$ ). Only 2 cases of squamous cell carcinoma were poorly differentiated (4.88\%).

One poorly differentiated squamous cell carcinoma case had areas of basis squamoid picture and focal spindling. One moderately-differentiated squamous cell carcinoma case had basosquamous features. One of the oesophagectomy specimen had features of basaloid carcinoma characterised by proliferation of oval to round, large pleomorphic basaloid cells with open pale chromatin pattern, small nuclei and scant cytoplasm arranged in solid and cribriform lobules often showing central necrosis, nests of tumour cells show peripheral palisading. Most of the adenocarcinomas were well differentiated except for two, one was moderately differentiated and the other poorly differentiated (Fig. 1 and 2).

In Barrett's oesophagus histochemistry with Alcian blue, PAS was performed (Fig. 6 and 7). Immunohistochemistry was performed in 2 doubtful cases and the results are shown in Table No. VII.

\begin{tabular}{|c|c|c|c|c|}
\hline $\begin{array}{c}\text { Sl. } \\
\text { No. }\end{array}$ & Period & $\begin{array}{c}\text { Total No. of } \\
\text { Biopsies }\end{array}$ & $\begin{array}{c}\text { Oesophageal } \\
\text { Biopsies }\end{array}$ & $\%$ \\
\hline 1 & $\begin{array}{c}\text { Jan 2000- } \\
\text { Dec 2000 }\end{array}$ & 410 & 49 & $11.95 \%$ \\
\hline 2 & $\begin{array}{c}\text { Jan 2001- } \\
\text { Dec 2001 }\end{array}$ & 510 & 59 & $11.56 \%$ \\
\hline 3 & $\begin{array}{c}\text { Jan 2002- } \\
\text { Dec 2002 }\end{array}$ & 613 & 56 & $9.13 \%$ \\
\hline 4 & $\begin{array}{c}\text { Jan 2003- } \\
\text { Dec 2003 }\end{array}$ & 523 & 52 & $9.94 \%$ \\
\hline 5 & $\begin{array}{c}\text { Jan 2004- } \\
\text { Dec 2004 }\end{array}$ & 469 & 37 & $7.88 \%$ \\
\hline 6 & $\begin{array}{c}\text { Jan 2005- } \\
\text { Dec 2005 }\end{array}$ & 300 & 24 & $8.0 \%$ \\
\hline \multicolumn{5}{|c|}{ Average } \\
\hline \multicolumn{5}{|c|}{ Table I: Shows Total Number of Oesophageal } \\
\hline \multicolumn{5}{|c|}{ Biopsy Specimen } \\
\hline
\end{tabular}

\begin{tabular}{|c|c|c|c|c|}
\hline $\begin{array}{c}\text { Sl. } \\
\text { No. }\end{array}$ & Period & $\begin{array}{c}\text { Table No. } \\
\text { of } \\
\text { Specimens }\end{array}$ & $\begin{array}{c}\text { Oeso- } \\
\text { phageal } \\
\text { Specimens }\end{array}$ & $\%$ \\
\hline 1 & $\begin{array}{c}\text { Jan 2000- } \\
\text { Dec 2000 }\end{array}$ & 2124 & 4 & $0.188 \%$ \\
\hline 2 & Jan-Dec 2001 & 2306 & 6 & $0.260 \%$ \\
\hline 3 & Jan-Dec 2002 & 2609 & 6 & $0.229 \%$ \\
\hline 4 & Jan-Dec 2003 & 2344 & 11 & $0.469 \%$ \\
\hline 5 & Jan-Dec 2004 & 2795 & 16 & $0.572 \%$ \\
\hline 6 & Jan-Dec 2005 & 2280 & 3 & $0.131 \%$ \\
\hline & \multicolumn{3}{|c|}{ Average } & $0.308 \%$ \\
\hline
\end{tabular}

Table IA: Shows the Incidence of Oesophageal Specimens

\begin{tabular}{|c|c|c|c|c|c|}
\hline \multirow{2}{*}{$\begin{array}{c}\text { Sl. } \\
\text { No. }\end{array}$} & \multirow{2}{*}{ Age Group } & \multicolumn{3}{|c|}{ No. of Cases } & \multirow{2}{*}{ \% } \\
\cline { 3 - 5 } & & $\mathbf{M}$ & $\mathbf{F}$ & $\mathbf{T}$ & \\
\hline 1 & $20-30$ yrs. & 9 & 6 & 15 & $5.415 \%$ \\
\hline 2 & $31-40$ yrs. & 19 & 18 & 37 & $13.357 \%$ \\
\hline 3 & $41-50$ yrs. & 42 & 26 & 68 & $24.548 \%$ \\
\hline 4 & $51-60$ yrs. & 73 & 19 & 92 & $33.2129 \%$ \\
\hline 5 & $61-70$ yrs. & 43 & 11 & 54 & $19.495 \%$ \\
\hline 6 & $71-80$ yrs. & 7 & 4 & 11 & $3.97 \%$ \\
\hline
\end{tabular}

Table II: The Age Distribution of Oesophageal Biopsies

\begin{tabular}{|c|c|c|c|c|c|}
\hline \multirow{2}{*}{ Sl. No. } & \multirow{2}{*}{ Age Group } & \multicolumn{3}{|c|}{ No. of Cases } & \multirow{2}{*}{$\%$} \\
\cline { 3 - 5 } & & $\mathbf{M}$ & $\mathbf{F}$ & $\mathbf{T}$ & \\
\hline 1 & $20-30$ & 9 & 0 & 1 & $2.173 \%$ \\
\hline 2 & $31-40$ & 0 & 3 & 3 & $6.521 \%$ \\
\hline 3 & $41-50$ & 6 & 6 & 12 & $26.086 \%$ \\
\hline 4 & $51-60$ & 14 & 5 & 19 & $41.304 \%$ \\
\hline 5 & $61-70$ & 8 & 2 & 10 & $21.739 \%$ \\
\hline 6 & $71-80$ & 1 & 0 & 1 & $2.173 \%$ \\
\hline Table IIA: The Age Distribution of Oesophageal Specimen \\
\hline
\end{tabular}




\begin{tabular}{|c|c|c|c|}
\hline Sl. No. & Features & Number of Cases & $\%$ \\
\hline 1 & Dyspepsia & 69 & $24.91 \%$ \\
\hline 2 & Dysphagia & 250 & $90.25 \%$ \\
\hline 3 & Loss of weight & 194 & $70.04 \%$ \\
\hline 4 & Anorexia & 152 & $54.87 \%$ \\
\hline 5 & Vomiting & 69 & $24.91 \%$ \\
\hline 6 & Odynophagia & 78 & $28.16 \%$ \\
\hline 7 & Regurgitation & 90 & $32.49 \%$ \\
\hline 8 & Hoarseness of voice & 2 & $0.72 \%$ \\
\hline 9 & Heart burn & 52 & $18.77 \%$ \\
\hline \multicolumn{4}{|c|}{$\begin{array}{c}\text { Table III: Shows the Distribution of Cases According to } \\
\text { their Clinical Presentation in Endoscopic Biopsies }\end{array}$} \\
\hline
\end{tabular}

\begin{tabular}{|c|c|c|c|c|c|}
\hline $\begin{array}{c}\text { Sl. } \\
\text { No. }\end{array}$ & Type of Lesions & M & F & Total & $\%$ \\
\hline \multirow{2}{*}{1} & Non-Neoplastic Lesions Normal & 11 & 3 & 14 & $0.05 \%$ \\
\cline { 2 - 6 } & No tissue & 11 & 3 & 14 & $5.05 \%$ \\
\hline 2 & Barrett's Oesophagus & 7 & 2 & 9 & $3.25 \%$ \\
\hline \multirow{3}{*}{3} & $\begin{array}{c}\text { Neoplastic-Benign Squamous } \\
\text { Papilloma }\end{array}$ & 1 & 0 & 1 & $0.36 \%$ \\
\cline { 2 - 6 } & Fibromuscular polyp & 1 & 0 & 1 & $0.36 \%$ \\
\hline 4 & Pre-Neoplastic SIL & 27 & 15 & 42 & $15.16 \%$ \\
\hline \multirow{4}{*}{5} & $\begin{array}{c}\text { Malignant Neoplasm Squamous } \\
\text { Cell Carcinoma - Usual Type }\end{array}$ & 115 & 60 & 175 & $63.18 \%$ \\
\cline { 2 - 6 } & Sarcomatoid carcinoma & 1 & 0 & 1 & $0.36 \%$ \\
\cline { 2 - 6 } & Adenocarcinoma & 16 & 2 & 18 & $6.49 \%$ \\
\cline { 2 - 6 } & Adenosquamous carcinoma & 1 & 0 & 1 & $0.36 \%$ \\
\cline { 2 - 6 } & Malignant Melanoma & 0 & 1 & 1 & $0.36 \%$ \\
\hline \multicolumn{5}{|c|}{$\begin{array}{c}\text { Table IV: Shows the Incidence of Lesions } \\
\text { and its Percentage e }\end{array}$} \\
\hline
\end{tabular}

\begin{tabular}{|c|c|c|c|}
\hline $\begin{array}{c}\text { Sl. } \\
\text { No. }\end{array}$ & Type of Lesions & No. of Cases & \% \\
\hline 1 & Barrett's Oesophagus & 7 & $2.527 \%$ \\
\hline 2 & SIL & 27 & $9.75 \%$ \\
\hline 3 & SCC & 116 & $41.877 \%$ \\
\hline 4 & Adenocarcinoma & 16 & $5.776 \%$ \\
\hline \multicolumn{3}{|c|}{ Table IVA: Incidence in Males } \\
\hline
\end{tabular}

\begin{tabular}{|c|c|c|c|}
\hline $\begin{array}{r}\text { Sl. } \\
\text { No. }\end{array}$ & Type of Lesions & No. of Cases & \% \\
\hline 1 & Barrett's Oesophagus & 2 & $0.72 \%$ \\
\hline 2 & SIL & 15 & $5.415 \%$ \\
\hline 3 & SCC & 60 & $21.66 \%$ \\
\hline 4 & Adenocarcinoma & 2 & $0.722 \%$ \\
\hline \multicolumn{3}{|c|}{ Table IVB: Incidence in Females } \\
\hline
\end{tabular}

\begin{tabular}{|c|c|c|c|}
\hline $\begin{array}{c}\text { Sl. } \\
\text { No. }\end{array}$ & Type of Growth & No. of Cases & \% \\
\hline 1 & Proliferative/Fungating & 39 & $84.78 \%$ \\
\hline 2 & Ulcerative & 4 & $8.69 \%$ \\
\hline 3 & Infiltrative & 3 & $6.52 \%$ \\
\hline \multicolumn{3}{|c|}{ Table V: Shows the Distribution of Cases According to the } \\
Morphological Appearance \\
\hline
\end{tabular}

\begin{tabular}{|c|c|c|c|c|c|c|c|}
\hline $\begin{array}{c}\text { Sl. } \\
\text { No. }\end{array}$ & Site & $\begin{array}{c}\text { No. of } \\
\text { Male } \\
\text { Cases }\end{array}$ & $\%$ & $\begin{array}{c}\text { No. of } \\
\text { Female } \\
\text { Cases }\end{array}$ & $\%$ & Total & $\%$ \\
\hline 1 & $\begin{array}{c}\text { Upper } \\
1 / 3 \mathrm{rd}\end{array}$ & 2 & $4.35 \%$ & - & - & 2 & $4.35 \%$ \\
\hline 2 & $\begin{array}{c}\text { Middle } \\
1 / 3 \mathrm{rd}\end{array}$ & 4 & $8.69 \%$ & 1 & $2.17 \%$ & 5 & $10.86 \%$ \\
\hline 3 & $\begin{array}{c}\text { Lower } \\
1 / 3 \mathrm{rd}\end{array}$ & 19 & $41.305 \%$ & 15 & $32.60 \%$ & 34 & $73.91 \%$ \\
\hline \multicolumn{7}{|c|}{ Table VA: Relative Distribution of Squamous Cell } \\
Carcinoma According to Site \\
\hline
\end{tabular}

\begin{tabular}{|c|l|c|}
\hline Male & Number of cases 25 & $60.97 \%$ \\
\hline Female & Number of cases 16 & $39.03 \%$ \\
\hline \multicolumn{2}{|c|}{ Table VB: Shows the Relative Distribution of } \\
Squamous Cell Carcinoma in Both Sexes \\
\hline
\end{tabular}

\begin{tabular}{|c|c|c|c|c|c|}
\hline $\begin{array}{c}\text { Sl. } \\
\text { No. }\end{array}$ & $\begin{array}{c}\text { Degree } \\
\text { Differentiation }\end{array}$ & No. of Cases & Total & Female & Percentage \\
\hline 1 & $\begin{array}{c}\text { Well-differentiated } \\
\text { SCC }\end{array}$ & 19 & 7 & 26 & $63.41 \%$ \\
\hline 2 & $\begin{array}{c}\text { Moderately- } \\
\text { differentiated }\end{array}$ & 8 & 5 & 13 & $31.707 \%$ \\
\hline 3 & $\begin{array}{c}\text { Poorly-differentiated } \\
\text { SCC }\end{array}$ & 2 & 0 & 2 & $4.88 \%$ \\
\hline \multicolumn{7}{|c|}{$\begin{array}{r}\text { Table VC: Shows the Relative Distribution of Squamous } \\
\text { Cell Carcinoma According to Differentiation }\end{array}$} \\
\hline
\end{tabular}

\begin{tabular}{|c|c|c|c|c|c|}
\hline Sl. & $\begin{array}{c}\text { Degree of } \\
\text { No. }\end{array}$ & Differentiation & Male. of Cases & Total & $\%$ \\
\hline 1 & $\begin{array}{c}\text { Well-differentiated } \\
\text { Adenocarcinoma }\end{array}$ & 3 & 0 & 3 & $60 \%$ \\
\hline 2 & $\begin{array}{c}\text { Moderately-differentiated } \\
\text { Adenocarcinoma }\end{array}$ & 1 & 0 & 1 & $20 \%$ \\
\hline 3 & $\begin{array}{c}\text { Poorly-differentiated } \\
\text { Adenocarcinoma }\end{array}$ & 1 & 0 & 1 & $20 \%$ \\
\hline \multicolumn{5}{|c|}{ Table VI: Shows the Relative Distribution of } \\
Adenocarcinoma According to Differentiation
\end{tabular}

\begin{tabular}{|c|c|c|c|c|c|}
\hline $\begin{array}{c}\text { Sl. } \\
\text { No. }\end{array}$ & Cases & S100 & Vimentin & Cytokeratin & EMA \\
\hline 1 & $\begin{array}{c}\text { Sarcomatoid } \\
\text { Carcinoma }\end{array}$ & - & + & - & - \\
\hline 2 & $\begin{array}{c}\text { Malignant } \\
\text { Carcinoma }\end{array}$ & + & - & - & - \\
\hline \multicolumn{6}{|c|}{ Table VII: Immunohistochemistry Results } \\
\hline
\end{tabular}

\begin{tabular}{|c|c|c|c|c|c|c|}
\hline \multirow{2}{*}{$\begin{array}{l}\text { Sl. } \\
\text { No. }\end{array}$} & \multirow{2}{*}{ Year } & \multirow{2}{*}{$\begin{array}{l}\text { Total No } \\
\text { pf Cancer } \\
\text { Patients }\end{array}$} & \multicolumn{3}{|c|}{$\begin{array}{c}\text { Oesophageal } \\
\text { Carcinoma }\end{array}$} & \multirow[t]{2}{*}{$\%$} \\
\hline & & & Male & Female & Total & \\
\hline 1 & $\begin{array}{c}\text { Jan 2000-Dec } \\
2000\end{array}$ & 397 & 37 & 4 & 41 & $10.327 \%$ \\
\hline 2 & $\begin{array}{c}\text { Jan 2000-Dec } \\
2000\end{array}$ & 426 & 36 & 6 & 42 & $9.859 \%$ \\
\hline 3 & $\begin{array}{c}\text { Jan } 2000-\mathrm{Dec} \\
2000\end{array}$ & 511 & 47 & 6 & 53 & $10.371 \%$ \\
\hline 4 & $\begin{array}{c}\text { Jan 2000-Dec } \\
2000\end{array}$ & 423 & 32 & 11 & 43 & $10.165 \%$ \\
\hline 5 & $\begin{array}{c}\text { Jan 2000-Dec } \\
2000\end{array}$ & 385 & 24 & 16 & 40 & $10.389 \%$ \\
\hline 6 & $\begin{array}{c}\text { Jan } 2000-\text { Dec } \\
2000\end{array}$ & 320 & 18 & 3 & 21 & $6.562 \%$ \\
\hline
\end{tabular}

\begin{tabular}{|c|c|c|c|c|c|c|c|}
\hline \multirow{2}{*}{$\begin{array}{c}\text { Sl. } \\
\text { No. }\end{array}$} & \multirow{2}{*}{ Symptoms } & $\begin{array}{c}\text { Orringer } \\
\mathbf{( 1 0 0} \text { Cases) }\end{array}$ & $\begin{array}{c}\text { Srisram } \\
(\mathbf{8 2} \text { Cases })\end{array}$ & $\begin{array}{c}\text { Present } \\
\text { Study }\end{array}$ \\
\cline { 3 - 8 } & & $\mathbf{N o .}$ & $\mathbf{\%}$ & $\mathbf{N o .}$ & $\mathbf{\%}$ & No. & $\%$ \\
\hline 1 & Dysphagia & 90 & $90 \%$ & 82 & $100 \%$ & 250 & $90.20 \%$ \\
\hline 2 & Odynophagia & 50 & $50 \%$ & 26 & $31.7 \%$ & 78 & $28.16 \%$ \\
\hline 3 & Anorexia & 48 & $48 \%$ & 49 & $60 \%$ & 152 & $54.87 \%$ \\
\hline 4 & Regurgitation & 30 & $30 \%$ & 30 & $36.6 \%$ & 90 & $32.49 \%$ \\
\hline 5 & $\begin{array}{c}\text { Hoarseness of } \\
\text { Voice }\end{array}$ & 4 & $4 \%$ & 3 & $3.6 \%$ & 2 & $0.72 \%$ \\
\hline \multicolumn{8}{|c|}{ Table IX: Correlation with Other Study } \\
\hline
\end{tabular}




\begin{tabular}{|c|c|}
\hline Lesions & Distinguishing Features \\
\hline $\begin{array}{l}\text { Benign squamous } \\
\text { papilloma }\end{array}$ & $\begin{array}{l}\text { Bland epithelium covering } \\
\text { fibrovascular cores }\end{array}$ \\
\hline Fibrovascular polyp & $\begin{array}{l}\text { Pedunculated mass of fibrous (+/- } \\
\text { adipose) tissue found in upper } \\
\text { oesophagus }\end{array}$ \\
\hline $\begin{array}{l}\text { Inflammatory fibroid } \\
\text { polyp }\end{array}$ & $\begin{array}{l}\text { Vascular fibroblastic tissue with } \\
\text { mixed inflammation }\end{array}$ \\
\hline Submucosal tumours & $\begin{array}{l}\text { Leiomyoma, granular cell tumour } \\
\text { most common }\end{array}$ \\
\hline $\begin{array}{l}\text { alignant squamous cell } \\
\text { carcinoma }\end{array}$ & Especially verrucous variant \\
\hline Adenocarcinoma & Infrequently polypoid \\
\hline Sarcomatoid carcinoma & $\begin{array}{l}\text { Atypical spindle cell stroma } \\
\text { predominates }\end{array}$ \\
\hline Malignant melanoma & $\begin{array}{l}\text { Melanin pigmentation; junctional } \\
\text { changes }\end{array}$ \\
\hline Sarcomas & $\begin{array}{l}\text { Rare, distinguished from carcinoma } \\
\text { with spindle cell component }\end{array}$ \\
\hline Metastatic tumours & Occasionally produce polypoid mass \\
\hline \multicolumn{2}{|c|}{$\begin{array}{l}\text { Table X: Differential Diagnosis of Polypoid Oesophageal } \\
\text { Tumours }\end{array}$} \\
\hline
\end{tabular}

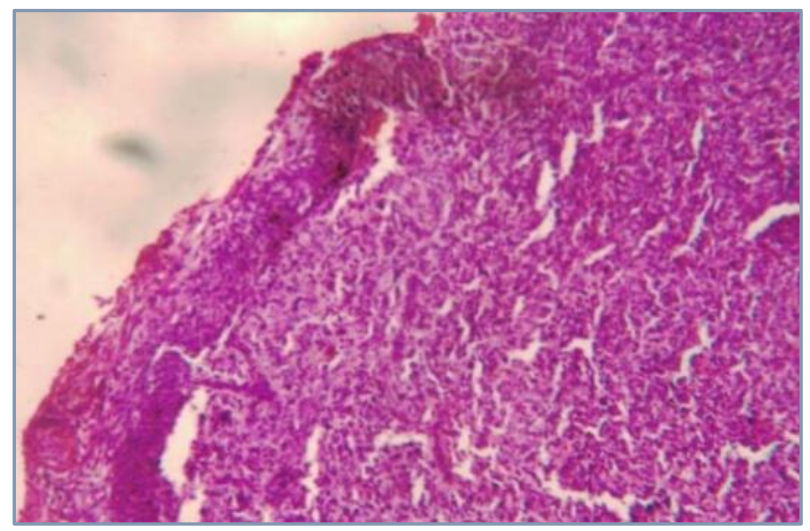

Fig. 3: Sarcomatoid Carcinoma - Epithelioid Malignant Squamous Cells Blend Imperceptibly with Sarcoma Component $\mathrm{H}$ and $\mathrm{E} X 100$

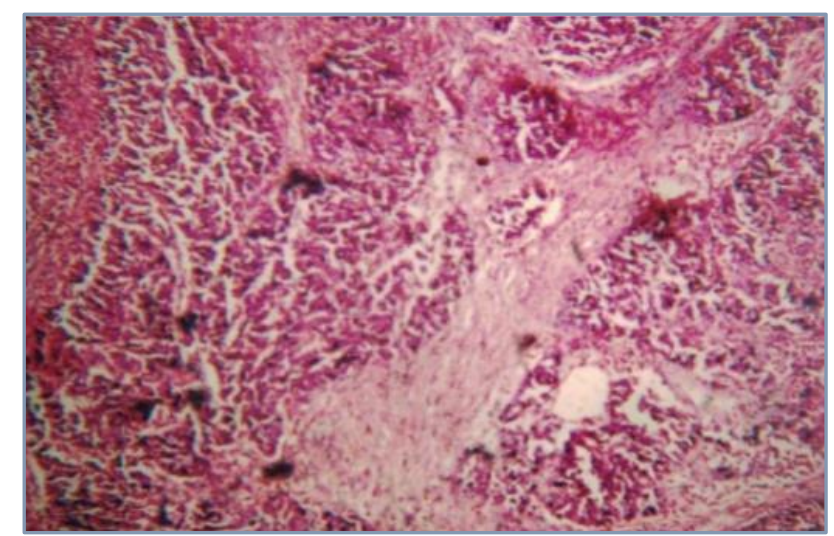

Fig. 1: Poorly-Differentiated Adenocarcinoma $\mathrm{H}$ and $\mathrm{E}$ X100

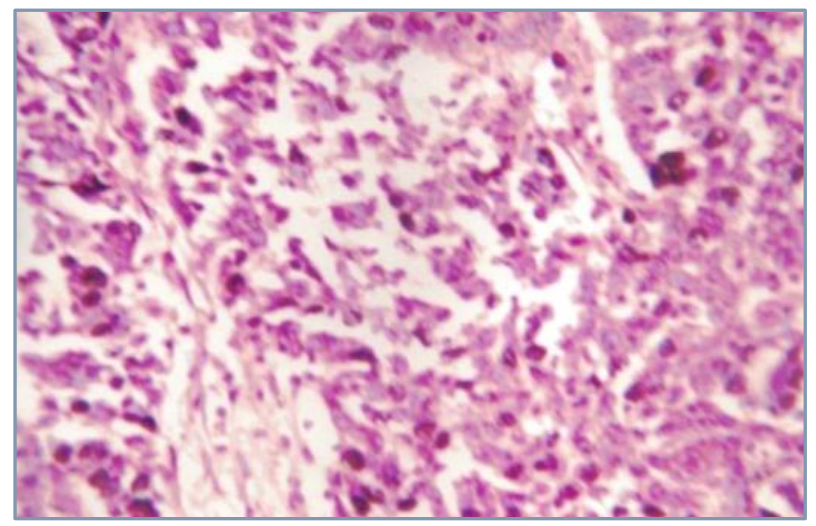

Fig. 2: High Power View of the Same H and E X400

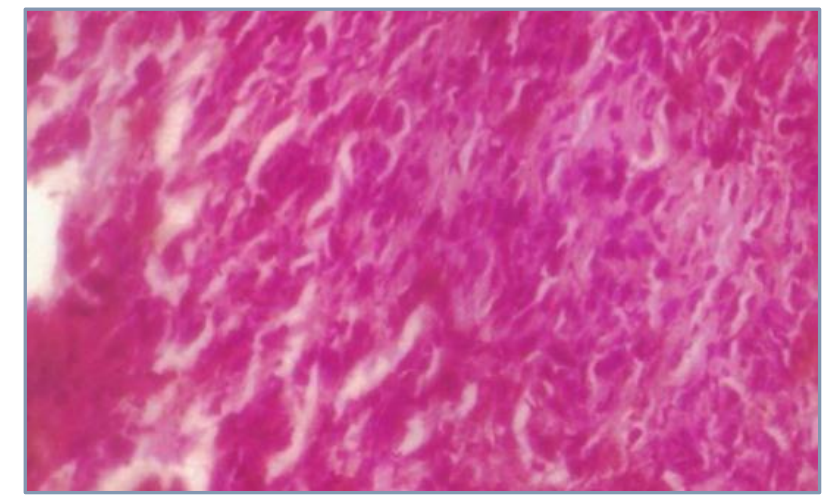

Fig. 4: High Power View of the Same -Spindled Cells X400

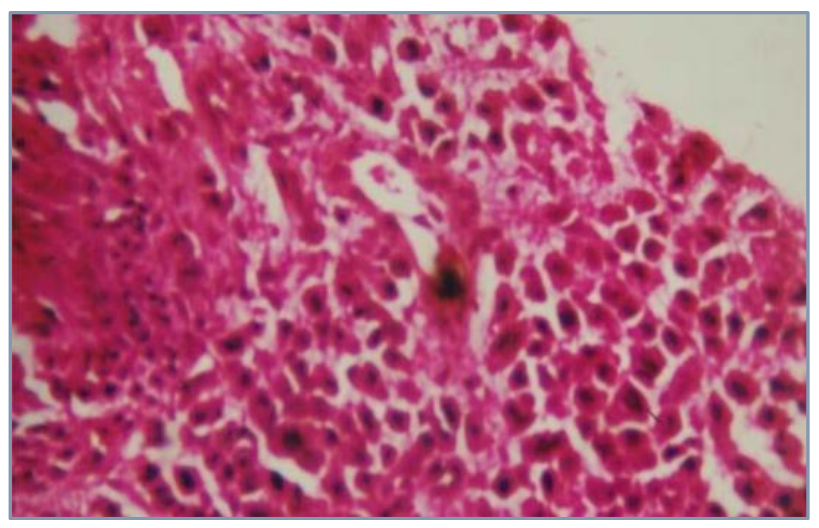

Fig. 5: Malignant Melanoma - Intracytoplasmic Pigmentation H and EX400

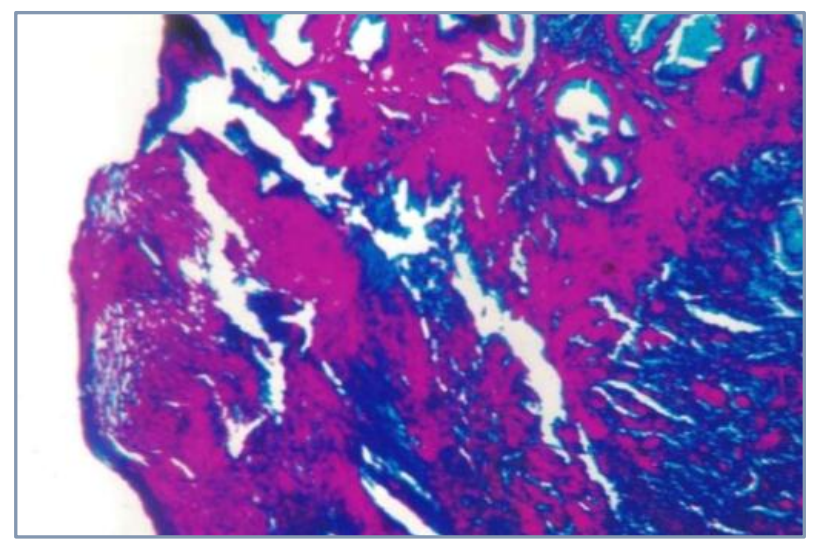

Fig. 6: Barrett's Oesophagus - Alcian Blue with PAS Positivity 


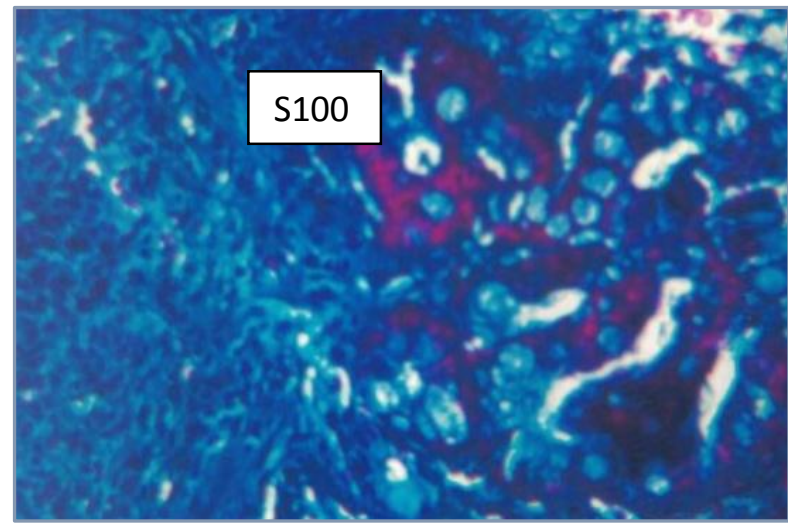

Fig. 7: High Power View of the Above X400. Scattered Intensely Blue Staining of Goblet Cells of Barrett's Metaplastic Epithelium

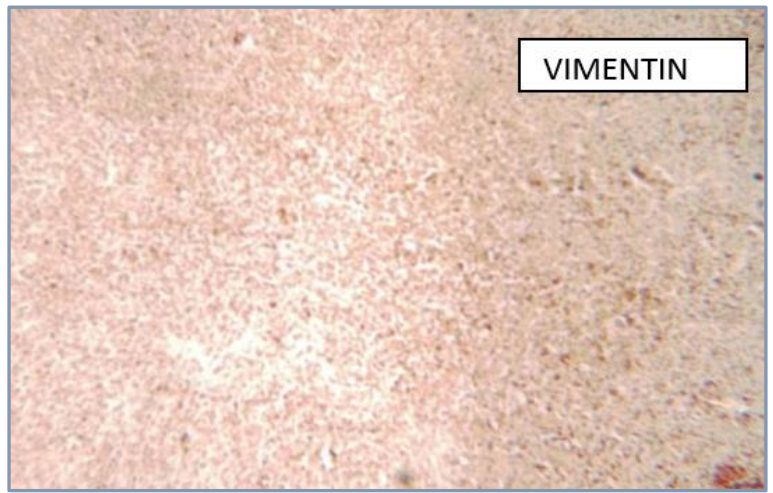

Fig. 8: Sarcomatoid Carcinoma shows Intense Vimentin Positivity X100

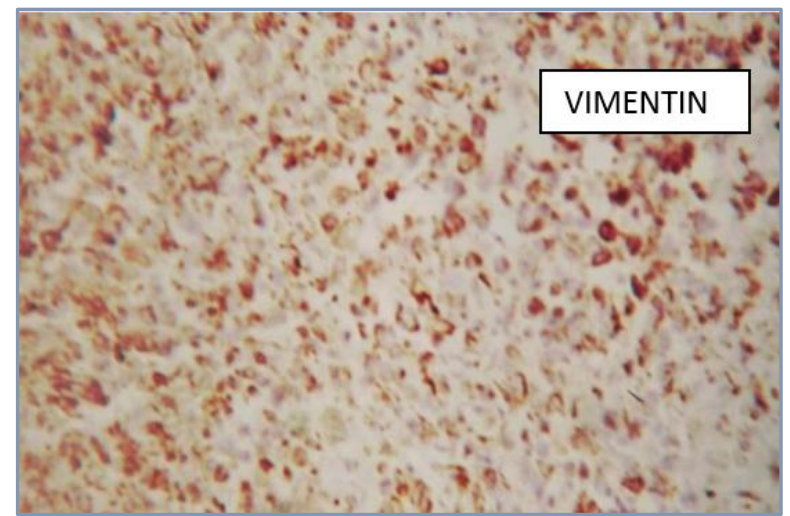

Fig. 9: High Power View of Above X400

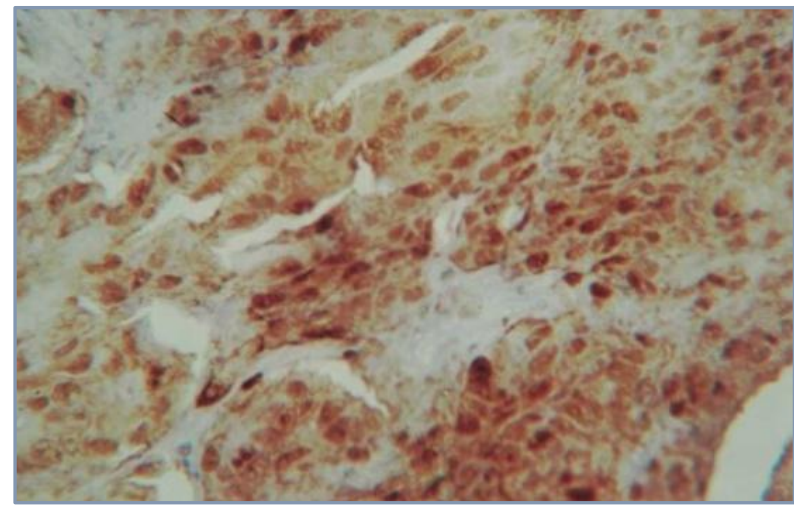

Fig. 10: Intense Nucleocytoplasmic Immunoreactivity for S100 Protein in Malignant Melanoma X400

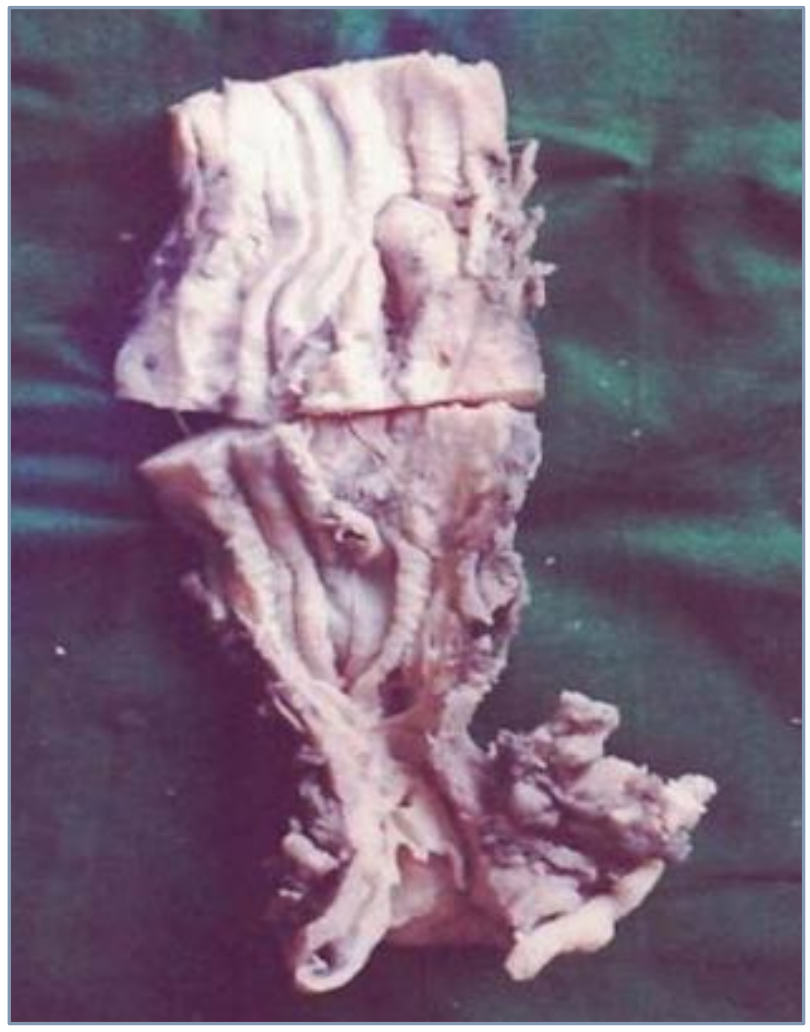

Fig. 11: Gross - Fungating Growth in Lower End of Oesophagus

\section{DISCUSSION}

The present study as in the others and literature shows that oesophageal lesions, particularly malignant neoplasms are one of the commonest cancers occurring in the world.1,2 Barrett's oesophagus as a result of GERD is now an accepted cause for further adenocarcinoma at lower end of oesophagus.3,4 The following Table No. VIII shows the incidence of oesophageal neoplasm in our study. Between January 2000 and September 2005, 2462 cancer patients attended out institution and of them 240 patients had oesophageal cancers, which constitutes $9.748 \%$ of the cancer registered in our hospital.

In the present study as in the others, the incidence of oesophageal lesions is high in the age group of 51-60 yrs. followed by 41-50 yrs. Das et al 1970 had also reported a high incidence of oesophageal cancers in this age group. In contrast, this age incidence is considerably lower than that reported in the Western countries, where it is common in sixties and seventies. In our study, oesophageal carcinomas occur more commonly in males with male-female ratio of $8: 1$ in contrast with figures of Registrar General of UK Statistics of 5:1.0.

In our study, $90 \%$ of cases complained of dysphagia and most of them had Grade III or Grade IV dysphagia. About 70\% of patients had loss of weight and anorexia followed by regurgitation of food in $33 \%$ of cases. The figures come in well correlation with clinical evaluation conducted by Orringer et al and Sriram et al in Thanjavur, March 2000 as shown in Table No. IX.

In our study 2 cases of well-differentiated squamous cell carcinoma, oesophagus is seen in $20 \mathrm{yrs}$. of age clearly shows the role of environment, food habits, nutrition and stress in the emergence of malignant neoplasm at the younger age group. Oesophageal cancer is modulated by not one single factor, but rather a large number of factors ${ }^{5}$ such as smoking, alcohol drinking and food consumption. The particular contributions 
of these factors to oesophageal cancer may differ among areas or countries along with variation in exposure levels both individually and in combination. Smoking has been proved to be a major risk factor for several sites of cancer such as nasal, oral and lung cancers. Ethanol is not a carcinogen itself, but may promote carcinogenesis.

Furthermore eating food rapidly increased the risk, while frequent consumption of fresh fruit, fresh vegetables and eggs was associated with protection.

Nutritional factors have been implicated in the aetiology of oesophageal cancer. Evidence from epidemiological and experimental studies suggest that deficiency of micronutrients namely vitamin $A$, zinc, selenium and magnesium are associated with oesophageal cancer. ${ }^{6}$

Risk factor ${ }^{7}$ for squamous cell carcinoma of the oesophageal have been identified such as tobacco, alcoholism, malnutrition and infection with human papilloma virus. The risk factors associated with oesophageal adenocarcinoma are less well defined.

The most important epidemiological difference between squamous cell carcinoma and adenocarcinoma, however, is the strong association between gastro-oesophageal reflux disease and adenocarcinoma. The results of a population-based controlled study suggest that symptomatic gastrooesophageal reflux is a risk factor for oesophageal adenocarcinoma. The frequency, severity and duration of reflux symptoms were positively associated with increased risk of adenocarcinoma. ${ }^{8}$

Barrett's oesophagus is noted in $3 \%$ to $12 \%$ patients with symptomatic GERD, who underwent endoscopy with biopsy. The definition of Barrett's oesophagus has been refined over the past 10 years. However, more recent studies have found that only those patients with intestinal metaplasia defined by the presence of acid mucin-containing goblet cells are at increased risk of developing dysplasia and adenocarcinoma and as such the definition of Barrett's oesophagus has now become dependent upon identification of goblet cells. Recently, the American College of Gastroenterology and its Practice Parameters Committee provided a definition of Barrett's oesophagus "as a change in the oesophageal epithelium of any length that can be recognised at endoscopy and is confirmed to have intestinal metaplasia by biopsy." Thus, the presence of goblet cells has become the accepted diagnostic criterion of this disease regardless of the precise and of biopsy within the tubular oesophagus.

In general, BE results from severe oesophageal mucosal injury by chronic gastro-oesophageal reflux disease interest in BE mainly stems from its well-established association with adenocarcinoma of the oesophagus.

The diagnosis of BE is readily achieved in patients who have long segments of IM by correlating endoscopic and histological findings. ${ }^{9,10}$ To avoid overdiagnosis of BE, its presence is considered to be established only if endoscopic examination reveals a displacement of the squamocolumnar junction and if IM is detected by histological examination.

A large body of research over the past decade has outlined many of the genetic changes that are important in the metaplastic-dysplastic carcinoma progression of oesophageal adenocarcinoma.11,12 Implicated genes including those involved in cell signalling, cell cycle control, cell adhesion and apoptosis are well characterised and their expression levels are known to be altered in oesophageal carcinogenesis.
Not only the history of oesophageal carcinoma, but also risk factors for developing of such cancer could differ overtime and from one region to another. Squamous cell carcinoma of the oesophagus in the developed world is at least five times more common in men than in women properly, because men drink and smoke more.

A diversity of benign tumours and non-neoplastic masses can be seen in the oesophagus. They are however mostly uncommon lesions, small and asymptomatic whose importance lies in their distinction from malignant tumours. Most clinically apparent tumours grow or protrude into the lumen and thus appears as polyps at endoscopy. The differential diagnosis of polypoid oesophageal lesions is summarised in following Table No. X.

In our study, 81 cases of non-cancerous lesions is observed in which 42 cases are with Squamous Intraepithelial Neoplasm (SIL) changes and 14 cases show only necrotic material/no tissue $(5.05 \%)$. There are rare lesions noted in less than $0.04 \%$ of endoscopic examination and typically represent an incidental finding in asymptomatic patients, although larger lesions cause dysphagia.

One case of fibromuscular polyp with intact overlying mucosa and core mature fibrous tissue exhibiting foci of myxoid areas and thin-walled vessels is seen.

Squamous cell carcinoma is the most common malignant tumour of oesophagus worldwide. It affects predominantly men with peak incidence in $5^{\text {th }}$ decade of life. Its incidence differs dramatically however in different countries with smoking, alcohol abuse, dietary exposure, genetic factors and HPV, all suspected of playing aetiological role.

The recognition of oesophageal malignancy in mucosal biopsy specimen is usually straight forward; 176 cases of squamous cell carcinoma and 18 cases of adenocarcinoma initially diagnosed with endoscopic biopsy evaluation and the ratio between squamous cell carcinoma and adenocarcinoma is 8.2:1. This figures come in correlation with studies conducted by various authors in the developing countries, especially in south Asian countries. But, in contrast, the studies conducted by Western people show an increased incidence of adenocarcinoma almost with 1:1 ratio probably attributed to their changes in lifestyle, increased intake of alcohol, smoking, food habits, etc. But our study correlates well with both South Asians as well as Western research workers that both in SCC and adenocarcinoma, the incidence is more common in male.

Squamous cell carcinoma are usually located in the lower and mid-oesophagus. Only about $10 \%$ are found in cervical and upper thoracic region.13,14,15 In accordance, in our study, only $4.35 \%$ are found in cervical and $10.86 \%$ are found in middle and $73.91 \%$ are found in lower oesophagus. At the time of diagnosis, most are advanced lesions with invasion into or beyond the muscularis propria. In our study, only one oesophagectomy specimen revealed adjacent nodal metastasis. Grossly, SCC usually present as fungating proliferative mass. In our study also 39 cases (84.78\%) presented as fungating proliferative mass.

One case of sarcomatoid carcinoma with biphasic histology exhibiting both carcinomatous and spindle cell components on immunohistochemistry revealed features of sarcoma with vimentin positivity (Fig. 8 and 9). One case of basaloid squamous cell carcinoma with characteristic lobules of basaloid cells with peripheral palisading, hyalinised basal lamina stroma, which is of poor prognosis was seen. ${ }^{16}$ 
One case of adenosquamous carcinoma characterised by mixed glandular and squamous differentiation is a rare aggressive neoplasm.

One case of primary malignant melanoma, which on immunohistochemistry showed positivity for S100 (Fig. 10). Most adenocarcinomas develop in the lower-third of oesophagus, some may extend into the proximal stomach.

\section{CONCLUSION}

The present study comprising of 277 oesophageal biopsies and 46 oesophagectomy specimens suggest the following conclusions.

1. The incidence of oesophageal lesions is $9.75 \%$.

2. Oesophageal lesions are common in males with male:female ratio of $2.2: 1$

3. Oesophageal lesions, both neoplastic and non-neoplastic are common in the lower end of oesophagus.

4. The habit of tobacco chewing along with betel nuts and leaves and consuming alcohol is considered to be major predisposing factor in our region.

5. Non-neoplastic oesophageal lesions are more prevalent in 31-40 years of age group.

6. Neoplastic oesophageal lesions are more prevalent in 5160 yrs. of age group.

7. Most common presenting symptom is dysphagia observed in all the patients under study followed by loss of weight and anorexia.

8. Most of the cancers are of squamous cell origin.

9. Barrett's oesophagus is noted in $3.52 \%$ of patients with symptomatic GERD, who undergo endoscopy with biopsy.

10. Barrett's oesophagus with dysplasia progress to adenocarcinoma.

11. Immunohistochemistry remains in its place as final confirmative tool in doubtful cases.

\section{REFERENCES}

1. Montgomery EA. Oesophageal cancer. World Cancer Report. World Health Organisation 2014:528-43.

2. Zhang, HZ, Jin GF, Shen, HB. Epidemiologic differences in oesophageal cancer between Asian and Western populations. Chinese Journal of Cancer 2012;31(6):281-6. doi:10.5732/cjc.011.10390.

3. Milne ANA, Carvalho R, Van Rees BPV, et al. Do collision tumours of the gastroesophageal junction exist? A molecular analysis. American Journal of Surgical Pathology 2004;28(11):1492-8.

4. Zuberi BF, Faisal N, Quarishy MS, et al. Correlation between clinical, endoscopic, and histological findings at oesophagogastric junction in patients of gastroesophageal reflux disease. JCPSP 2005;15(12): 774-7.
5. Prabhu A, Obi KO, Rubenstein JH. The synergistic effects of alcohol and tobacco consumption on the risk of oesophageal squamous cell carcinoma: a meta-analysis. The American Journal of Gastroenterology 2014;109(6):822-7. doi:10.1038/ajg.2014.71.

6. Jimenez P, Piazuelo E, Sanchez MT, et al. Free radicals and antioxidant systems in reflux oesophagitis and Barrett's oesophagus. World J Gastroenterol 2005;11(18):2697703.

7. Chun-Xia Y, Hua-Yu W, Zhi-Ming W, et al. Risk factors for oesophageal cancer a case-control study in south-western China Asian Pacific. Asian Pac Journal of Cancer Prevention 2005;6(1):48-53.

8. Takubo K, Vieth M, Honma N, et al. Ciliated surface in the oesophagogastric junction zone: a precursor of Barrett's mucosa or ciliated pseudostratified metaplasia. American Journal of Surgery Pathology 2005;29(2):211-7.

9. Takubo K, Vieth M, Aryal G, et al. Islands of squamous epithelium and their surrounding mucosa in columnarlined oesophagus: a pathognomonic feature of Barrett's oesophagus? Human Pathology 2005;36(3):269-74.

10. Abnet CC, Huppi K, Carrera A, et al. Control region mutations and the common deletion are frequent in the mitochondrial DNA of patients with oesophageal squamous cell carcinoma. BMC Cancer 2004;4:30.

11. Kwong D, Lam A, Guan X, et al. Chromosomal aberrations in oesophageal squamous cell carcinoma among Chinese: gian of $12 p$ predicts poor prognosis after surgery. Human Pathology 2004;35(3):309-6.

12. Faundes RB, Mello CR, Tollens $\mathrm{P}$, et al. p53 protein in oesophageal mucosa in individuals at high risk of squamous cell carcinoma of the oesophagus. Diseases of the Oesophagus 2001;14(3-4):185-90.

13. Csendes A, Smok G, Flores N, et al. Comparison of clinical endoscopic and functional findings in patients with intestinal metaplasia at the cardia, carditis, and shortsegment columnar epithelium of the distal oesophagus with and without intestinal metaplasia. Diseases of the Oesophagus 2000;13(1):61-8.

14. Hirota WK, Loughney TM, Lazas DJ, et al. Specialised intestinal metaplasia, dysplasia, and cancer of the oesophagus and oesophagogastric junction: prevalence and clinical data. Gastroenterology 1999;116(2):277-85.

15. Koide N, Nishio A, Kono T, et al. Histochemical study of angiogenesis in basaloid squamous carcinoma of the oesophagus. Diseases of the Oesophagus 2000;13(2): 142-7.

16. Gadour MO, Ayolla EA. Primary malignant melanoma of the oesophagus: a case report and review. Tropical Gastroenterology 2000;21:185-7. 